\title{
LA INTERCULTURALIDAD HOY, COMO TENDENCIA EDUCATIVA
}

\author{
Marcela Chavarría Olarte
}

\section{RESUMEN}

Hoy, la educación está matizada por múltiples tendencias, producto del sorprendente avance de la ciencia y la tecnología, la reducción de tiempos y distancias en los procesos de comunicación y la globalización cultural. El reto pedagógico es, pues, educar "para la vida" en un mundo globalizado, formar en la interculturalidad que acerque a los pueblos y a las personas en el encuentro con distintas formas de expresión de unos mismos valores. El presente escrito guía la reflexión pedagógica en torno a los signos que caracterizan los primeros años del nuevo siglo y milenio; se pregunta sobre el papel de los educadores en esta etapa de la Historia; plantea la necesidad de formar en habilidades del pensamiento y la acción para forjar y afrontar escenarios futuros; describe seis tendencias educativas presentes a nivel internacional; y esboza algunos rasgos que han de caracterizar a un modelo educativo de calidad.

\section{INTRODUCCIÓN}

La educación actual está matizada por múltiples tendencias. La sorprendente evolución de la tecnología motiva importantes cambios en las "formas" de planear, realizar y evaluar los procesos educativos. Las distancias se acortan y el acceso a la información se amplía y simplifica en grandes proporciones. Todo ello nos induce a educar "para la vida" en un mundo globalizado, a formar en la interculturalidad, que acerque a los pueblos y a las personas en el encuentro con diferentes formas de expresión de unos mismos valores.

¿Cuál es el papel de los educadores en esta etapa de la Historia..? 


\subsection{LAS GRANDES TENDENCIAS DE LA EDUCACIÓN}

Una tendencia educativa es una corriente de pensamiento y de acción para la formación del ser humano; un comportamiento estable y generalizado hacia el cual nos dirigimos; la dirección hacia donde vamos, el conjunto de aspiraciones que obedecen a los signos de los tiempos y a las posibilidades para obrar en algún campo, en este caso, en el educativo.

Esas aspiraciones hacia las cuales tiende la educación, son globales, se visualizan como un denominador común en el ámbito mundial, en función de los signos de los tiempos a inicio del tercer milenio.

El siglo XX se caracterizó por avances vertiginosos en la ciencia y la tecnología; avances tan acelerados que sorprendieron fuertemente a padres y maestros sin estar preparados para abordarlos y, a la humanidad entera, sin un avance paralelo en la reflexión ético-filosófica que permitiera integrarlos a la vida humana de modo gradual, equilibrado y positivo.

Especialmente en la segunda mitad del siglo XX, nos acostumbramos a crecer en un ambiente de sucesivos cambios y novedades, en donde la estabilidad de los valores se puso a prueba.

Viajes interplanetarios, invasión de la informática, tecnología genética, ingeniería biomédica, entre otros progresos, rompieron con la estabilidad de los sistemas educativos, obligando a dar un giro de $180^{\circ}$ en ellos: pasar del gis al "power point, del pupitre individual y rígido a las mesas colectivas y ensamblables, del libro de texto a Internet, de las carreras clásicas a la pluriespecialización; de la docencia centrada en la enseñanza del maestro al aprendizaje centrado en la interrelación del alumnado, etcétera. ¿Cuáles son los pautas clave de la sociedad, y hacia dónde apuntan las tendencias, frente a los retos del tercer milenio..?

Gerardo Castillo señala: "Un punto de referencia obligado y fundamental para indagar acerca de la educación del futuro son los retos actuales del cambio social para la educación. 
La respuesta a estos retos, desde la familia y desde los centros educativos, será en buena parte, la educación del futuro ${ }^{1}$.

\subsection{ALGUNOS SIGNOS DEL DIAGNÓSTICO EDUCATIVO ACTUAL}

¿Qué cambios se han operado en las últimas décadas en la familia, escuela y sociedad?

El doctor Salvador Cerón en su obra Un Modelo Educativo para México ${ }^{2}$, hace referencia a algunos "síntomas" del panorama educativo actual, que recaen en modelos educativos desgastados.

Es frecuente encontrarse con estilos educativos, de padres y maestros, que no responden a las necesidades actuales; patrones que se repiten de generación en generación relacionados con el qué y cómo de la educación, que al tener su origen en circunstancias pasadas, están muy lejos de preparar a los niños y jóvenes para enfrentar los actuales retos del mundo y para crear y desarrollar los del futuro.

Existen pues, modelos educativos desgastados en la familia y la escuela, que no responden a las necesidades actuales y futuras.

Hace apenas algunas décadas, existían planes y programas de estudio que podían mantenerse por años debido a la estabilidad del contexto cultural. Los hermanos mayores heredaban sus libros a los menores pues, por lapsos considerables, se mantenían los programas de estudio. Era claramente conocido el perfil de enseñanza-aprendizaje de cada grado de primaria y secundaria y claramente predecible el contenido de estudio y el campo de trabajo de las carreras clásicas: Derecho, Ingeniería Civil, Medicina, etcétera.

"Hoy se deja sentir la necesidad de un sistema de educación cuyo papel no sea solamente ponernos en condiciones

${ }^{1}$ CASTILLO, G., "La educación del futuro" en: AAVV, Lo Permanente y lo Cambiante en la Educación., p.16.

${ }^{2}$ Cfr. CERON, S., Un Modelo Educativo para México., p.25-38. 
de comprender un pasado relativamente estable y un presente inestable, papel que la escuela realizaba antes, por medio de un tipo de aprendizaje que los autores de este estudio llaman el "aprendizaje conservador"; lo que hace falta hoy, como lo preveía, hace más de diez años, la Comisión internacional de la UNESCO $^{3}$ para el desarrollo futuro de la educación, es un aprendizaje que sea, a la vez, anticipador y participativon"

El papel del maestro - o de los padres- y del alumno - o de los hijos-, estaba perfectamente diferenciado, proporcionando siempre al educador el papel protagónico en las decisiones y en las acciones. Los "libros de texto" eran el eje conceptual alrededor del cual giraba la enseñanza; la oferta de opciones educativas era relativamente corta y por tanto conocida por el común de la gente.

En las últimas décadas, el cambio se convirtió en la constante; al grado de que hoy, los programas de asignatura se construyen frecuentemente por maestros y alumnos a lo largo del año escolar a partir de sus expectativas e intereses, los libros de texto han caído en desuso para ser sustituidos por la "cultura de la fotocopia" que permite bajar de Internet, día a día, "las novedades" de la asignatura.

Los perfiles de ingreso y egreso de las distintas modalidades de estudio, se han hecho a tal grado flexibles, que es común entrar a la Universidad sin haber decidido qué carrera estudiar. En virtud de la abundancia de "troncos comunes de estudios", los perfiles de egreso son casi personales pues las "tiras de materias" se eligen en buena medida por los estudiantes; y los objetivos se centran hoy en el desarrollo de competencias (habilidades y actitudes personales), no ya en la acumulación de saberes cognitivos.

3 United Nations Educational, Scientific and Cultural Organization (Organización de las Naciones Unidas para la Educación, la Ciencia y la Cultura).

${ }^{4}$ ADISESHIAH, M., "Tendencias y perspectivas de la educación" en: AAVV, Sobre el Futuro de la Educación. Hacia el año 2000., p.48-49. (Cfr.: FAURE, E. y otros: "Aprender a ser". Alianza/Unesco, Madrid, 1973). 
El protagonista del proceso educativo es hoy el educando, y el educador ha pasado a ser su "facilitador", su "conductor", su "animador". "(...) los métodos o enfoques pedagógicos preferidos se han definido de formas diversas, como "aprendizaje mediante la experiencia", "aprendizaje mediante la investigación", "aprendizaje mediante el descubrimiento" y "aprendizaje en clase abierta", que se remontan a Dewey" ${ }^{5}$.

Finalmente, los campos de trabajo también se han diversificado y super especializado. Cada año se abren nuevas ofertas de estudio de pregrado y de posgrado; cada estudiante puede diseñar su perfil profesional y tiene, ante sí, el reto de abrir su propio campo laboral.

Por todo lo anterior: «La finalidad de la educación debe ser no sólo formar jóvenes con miras a un oficio determinado sino sobre todo capacitarlos para que puedan adaptarse a tareas diferentes y perfeccionarse sin cesar a medida que evolucionan las formas de producción y las condiciones de trabajo; así, la educación debe tender a facilitar la reconversión profesional ${ }^{6}$.

Siempre se ha tenido claro que el buen profesionista requiere actualización constante, pero hoy, los contenidos de estudios se hacen obsoletos antes de egresar de la Universidad. El título profesional que se recibe no representa ya, un conjunto de saberes que se domina para ser aplicados en situaciones conocidas, sino un conjunto de competencias profesionales para aplicarse a la solución de problemas variados en situaciones impredecibles.

Los curricula universitarios tienden a ser flexibles y multidisciplinarios. Existe un "interés por lograr una integración de campos de conocimiento y experiencia que faciliten una comprensión más reflexiva y crítica de la realidad, subrayando no sólo dimensiones centradas en contenidos culturales, sino también el dominio de los procesos que son necesarios para

${ }^{5}$ UNESCO., Informe Mundial sobre la Educación 1998: los Docentes y la Enseñanza en un Mundo de Mutación., p.93-94.

${ }^{6}$ UNESCO., 21 Puntos para una Nueva Estrategia de la Educación., p.18. 
conseguir alcanzar conocimientos concretos y, al mismo tiempo, la comprensión de cómo se elabora, produce y transforma el conocimiento, así como las dimensiones éticas inherentes a dicha tarea. Todo lo anterior subraya un objetivo educativo tan definitivo como es el "aprender a aprender"»?

\subsection{LOS EDUCADORES HOY}

- Ante este panorama, padres y profesores del "modelo tradicional» son fácilmente superados por sus educandos en la comprensión y solución de asuntos ordinarios. Es común que sean hoy los niños quienes enseñan a los adultos cómo accionar un aparato electrónico, cómo bajar de Internet una información deseada, cómo conducirse en un ambiente virtual, etcétera.

Es común que los padres no tengan la preparación necesaria para resolver las dudas y problemas escolares de sus hijos; lo cual les coloca en desventaja para el control que les corresponde en el proceso educativo y demerita su imagen de autoridad.

Muchos de nuestros abuelos nacieron, crecieron y murieron dentro del patrón de vida de su pueblo o ciudad. Sus padres podían prever casi con exactitud su estilo de vida, escolaridad y trabajo desde su nacimiento.

Hoy más que nunca los hijos son un reto ante un futuro incierto para el cual es preciso educarles.

¿Cómo guiar acertadamente a los hijos o alumnos, si no se tiene claro hacia dónde?

Si educamos con contenidos y formas que no responden a sus necesidades, el futuro de la sociedad está en crisis.

"La educación no responderá eficazmente a los retos del cambio si se limita a actuar desde lo que no cambia, desde lo permanente. Ello supondría desfase, parálisis, inadaptación. Pero tampoco tendrá éxito la educación si se guía solamente por lo cambiante. En este segundo caso habría pérdida de rumbo»" 
El educador actual, más que centrarse en la transmisión de una herencia del pasado, debe abocarse a la habilitación para forjar y afrontar escenarios futuros.

Los niños, adolescentes y jóvenes —educadores de hoynecesitan desarrollar habilidades de pensamiento y de acción, actitudes acertadas hacia la vida a través de modelos creativos e innovadores que les permitan saber qué hacer en situaciones variadas, dónde y cómo buscar la información requerida en cada momento, hábitos de trabajo y convivencia que les permitan colaborar en equipos de trabajo interdisciplinarios e interculturales.

Necesitan desarrollar: un pensamiento crítico sustentado en valores, una voluntad recia forjada en virtudes, y una afectividad encauzada hacia el amor verdadero.

\subsection{LA ÉTICA DEL PROCESO EDUCATIVO}

- Pero, no obstante los acelerados cambios en los contenidos y formas de enseñanza-aprendizaje, los modelos educativos van a un ritmo diferente al de evolución de la bumanidad. La ciencia y la técnica avanzan y se transforman a ritmo más veloz que la investigación pedagógica, psicopedagógica y humanística en general. Los modelos educativos van "por detrás", adaptando y corrigiendo sobre "la marcha".

La reflexión ética llega con frecuencia tarde ante los avances científicos y tecnológicos. Primero se introdujo en la sociedad la reproducción artificial y después se percató de los problemas éticos que planteaba. Los padres introducen sistemas televisivos multiculturales en el hogar, y tiempo más tarde descubren que "el enemigo" de sus esfuerzos por educar en valores está en casa y que tiene mucho mayor poder de persuasión que ellos. El Internet se introduce de modo casi obligado en nuestros hogares, y frecuentemente los padres o madres no saben usarlo y, por lo tanto, no pueden evaluar sus beneficios ni prevenir sus posibles perjuicios. 
Todo avance o conquista en la ciencia y la tecnología es por sí mismo positivo - en cuanto conocimiento del cosmos y dominio de las fuerzas y elementos naturales- pero el hecho de que sea positivo en sí mismo no implica, necesariamente, que sea positivo para la vida humana. Esto último requiere de la valoración anticipada de sus consecuencias inmediatas y mediatas, de una oportuna legislación a partir de consideraciones éticas, de un mesurado uso en función de objetivos controlables, etcétera.

«Proporcionar una orientación en cuanto a los valores es otra de las tareas de la educación en una sociedad en la cual la alienación es endémica y que está profundamente interesada por cosas, técnicas y procesos en los cuales los valores tienden a ser confusos y a crear conflictos".

Hace muchos años que, a consecuencia del cúmulo cada vez mayor de conocimientos, padecemos de la saturación de saberes en detrimento de la formación en valores. Padres y maestros se han preocupado más por lo que el niño o joven sabe que por lo que es como persona humana. La urbanidad, el civismo, la moral, la religión, fueron dejando paso al materialismo, al cientificismo, al hedonismo y a una larga lista de "ismos" producto de las ideologías que, a falta de ideas y convicciones firmes, se han ido instalando en la mente y el alma de niños y adultos.

Se ba confundido cantidad con calidad en la educación, enfatizando la instrucción masiva por encima de la educación personalizada; el adiestramiento y la tecnologización, por encima de la creatividad y el servicio social.

El trinomio saber-hacer-tener, que representa el avance de ciencia y tecnología, siendo positivo por sí mismo, ha dejado de serlo al trastocar el ser del hombre, representado por el respeto a la naturaleza humana, la eticidad en las relaciones personales, la primacía de las ciencias humanas como faro que guía el desarrollo de las ciencias exactas.

9 TABA, H., Elaboración del Currículo: Teoría y Práctica., p.68. 
Muchos profesores ya no reconocen como parte de su función magisterial la formación del carácter de los alumnos ni su educación en virtudes humanas. Se forma (o deforma) a los alumnos en la competitividad por encima de la ética. Ganar a como dé lugar, situarse por encima de otros, velar por la propia satisfacción independientemente de las necesidades ajenas. Esto ha dado por resultado "genios de la economía" que son capaces de transformar los rumbos del país pero para beneficio -ilícito por supuesto- de ellos mismos, o profesionistas de cualquier ramo que sólo conciben el ejercicio de su profesión como un medio de lucro, nunca de servicio.

La sociedad plantea hoy unas exigencias en las que no cabe la mediocridad. Por ello es preciso evaluar nuestros modelos educativos y contar con un "eje formativo" que ilumine el "perfil de egreso" de la familia y de la escuela en función de la concepción de la educación como un proceso de perfeccionamiento integral de la personalidad, en el cual la interculturalidad juega un papel muy importante.

\section{5. ¿CUÁLES SON LAS GRANDES TENDENCIAS EDUCATIVAS ACTUALES?}

Entre las corrientes de pensamiento y de acción que a nivel internacional están presentes hoy en los cambios y en la evolución de la educación, podemos distinguir:

\section{a) Avance acelerado de la tecnología e influencia de ésta en la vida diaria.}

En una reunión convocada por la UNESCO y el CEID (Asia-pacific Centre of Educational Innovation for Development) en Bangkok (diciembre de 1996), en la mesa redonda titulada "Una nueva concepción de la utilización de la tecnología educativa en los países en desarrollo", se comentó entre otros aspectos que:

"Las nuevas tecnologías planteaban problemas relacionados con: a) sus repercusiones en la cultura; b) su influencia en las comunidades; c) la autoridad y el control; d) la elaboración de 
los planes de estudios y el acceso a ellos; e) la naturaleza de la escuela, f) la competencia de los docentes; y g) la igualdad y la justicia social ${ }^{10}$.

Ciertamente el avance acelerado de la tecnología en los últimos años y su influencia en la vida diaria familiar, escolar y social, ha ocasionado, a la par de mayor eficiencia en los procesos industriales, la automatización de los procesos humanos y cambios profundos en la dinámica de la comunicación interpersonal.

Cada vez tenemos mayor contacto con máquinas y menor contacto con personas (audiobanco, "webmarket" ${ }^{11}$, autoservicios, etcétera).

En el ámbito escolar, las computadoras son un instrumento básico de enseñanza-aprendizaje desde el nivel preescolar. Los "café-Internet" proliferan como espacios de tareas escolares.

En el ámbito familiar la comida va dejando de tener «el sazón de la casa" para tener el de la marca de congelados que procesamos en microondas. Los padres, y hasta los hijos, suelen tener, $\boldsymbol{s} \boldsymbol{u}$ teléfono celular personal, $\boldsymbol{s u}$ televisión en la recámara y su correo electrónico individualizado por el que a veces es más fácil comunicarse entre sí que de modo personal

b) Globalización, en la información, en el mercado, y en la educación por supuesto.

Hace apenas unos cuantos años, revalidar en México estudios realizados en el extranjero era toda una proeza. Hoy forma parte de los currícula universitarios el intercambio académico internacional y las estancias de los estudiantes en el extranjero para acreditar parte de sus estudios en el propio país y cuando se trata de un posgrado, antes de traspasar la frontera, el estudiante cuenta con los convenios interinstitucionales que le validan sus estudios.

${ }^{10}$ UNESCO., Informe Mundial sobre la Educación 1998: los Docentes y la Enseñanza en un Mundo de Mutación., p.91.

${ }^{11}$ Servicios de compra-venta por Internet. 
"Más que crear un sistema de equivalencia educativa, la $\mathrm{OCDE}^{12}$ estudia la creación de un mercado abierto en el intercambio de valor educativo" ${ }^{13}$.

El "Bachillerato Internacional" se está generalizando en distintos países; y los estudiantes de todos los niveles educativos, "beben información" de los mismos servidores de Internet.

La "OCDE ha empezado a examinar los temas de reciprocidad en las credenciales y créditos académicos. Esto ha resultado en la identificación provisional de tres principios sobre el aseguramiento de la calidad en la educación internacional:

1. La transparencia implica que la información debe estar disponible para los varios depositarios.

2. La comparabilidad implica que dicha información puede ser presentada en formas que se relacionan con los sistemas y los intereses de los depositarios en distintos entornos nacionales y regionales.

3. La convertibilidad implica que existen "valores" específicos que pueden adjuntarse a la participación en actividades internacionales ${ }^{14}$.

Con todo lo positivo que implica lo anterior, existen algunas consideraciones importantes a hacer. Por ejemplo, el hecho de que son prioritariamente los llamados "países desarrollados" los que marcan la pauta de la información que se comparte, y los países "en vías de desarrollo" sufren la inculturación forzosa de esos contenidos, que en muchas ocasiones no se adaptan a su realidad nacional.

c) Comunicación en el ámbito mundial. Los lapsos de comunicación se han hecho cada vez más cortos. Hemos pasado del correo postal al fax, al correo electrónico, al diálogo inmediato por computadora.

${ }^{12}$ Organización para la Cooperación y el Desarrollo Económico.

${ }^{13}$ PALIN, P., "El contexto global para la garantía de la calidad en la Educación Superior: Actividades Recientes de la OCDE. Resumen" en: Reunión Trilateral sobre la Globalización de la Educación Superior y las Profesiones: La Globalización de la Educación Superior y las Profesiones: el Caso de América del Norte., p.193.

${ }^{14}$ Idem. 
Esto ha significado un invaluable apoyo a la comunicación y trabajo humanos, pero representa, a la vez, un riesgo de despersonalización.

¿Cuánta gente desarrolla hoy todo su trabajo en casa con la sola "Compañía" y apoyo de su computadora..? ¿Cuántas relaciones humanas —incluso matrimoniales — se construyen y se destruyen hoy por correo electrónico..? ¿La informática es un medio para el desarrollo del hombre, o el hombre un medio para el desarrollo de la informática..?

"Los mass-media, junto a grandes posibilidades relacionadas con la difusión de la información, tienen el riesgo de sofocar la capacidad de juicio propio de cada persona. Los juicios previamente elaborados pueden sustituir al juicio personal.

"El reto supone preparar a las personas, desde la infancia, para utilizar los mass-media con capacidad crítica. Para ello es preciso convertir estos medios en medios de autoeducación ${ }^{\prime 15}$.

d) Super especialización de saberes y funciones. La ciencia y la tecnología avanzan tan rápido, y se ha llegado a tanta gama de saberes, que el presente de la formación profesional se caracteriza por la super especialización.

Cada especialista sabe de lo suyo, y "lo suyo" es cada vez más delimitado y profundo. Cada profesional se convierte en un eslabón de un complejo engranaje de sujetos y acciones que deben —o deberán— hacerse en equipo para entender y resolver acertadamente los problemas que atiendan.

Por ejemplo, hace cincuenta años se acudía "al médico" para cualquier problema de salud. Se hablaba "del médico" (de todo) y de "la salud" (completa). Poco a poco hubo que acudir a diversos especialistas para diferentes padecimientos y hoy, hasta para un mismo padecimiento, interviene un grupo de diferentes especialistas.

Las carreras profesionales de ayer, hemos señalado, eran pocas y conocidas; las de hoy son innumerables y cambiantes; las del futuro próximo (las que estudiarán nuestros hijos hoy pequeños) las desconocemos porque no se han creado aún.

${ }^{15}$ CASTILlO, G., op. cit., p.21. 
"El reconocimiento de la necesidad suprema de la educación de brindar una perspectiva hacia la sociedad integral sugiere, además, el imperativo de fortalecer la educación general más que la especial. Quizás incluso todo el concepto de la función liberadora de las disciplinas especializadas tendría que ser reconsiderado. Un problema de importancia crucial es el modo de pensar fraccionado y lineal creado por la ejercitación especializada que ubica diferentes ideas en diferentes esferas de actividad. Puesto que los asuntos sociales y humanos importantes no pueden ser percibidos adecuadamente mediante este modo de pensar especializado, la educación necesita neutralizar el impacto de estas perspectivas especializadas. Ha de cultivar formas de pensamiento que sean más apropiadas para tratar problemas interdependientes y que siempre incluyen variables humanas. La educación debe ayudar al individuo a encontrar el sostén que la cultura le ha ayudado a perder ${ }^{16}$.

e) Prioridad de los valores materiales sobre los humanos. El tener y el saber por encima del ser... aunque éste último trastoque su naturaleza y muera. ¿Cuánto ganas es igual a cuánto vales?... "Time is money... todo esto produce una inversión en la jerarquía correcta de valores; un desfase entre las ciencias exactas y las ciencias humanas; un progresivo surgimiento de síntomas deshumanizadores: aborto, eutanasia, manipulación genética, clonación... ¿̇hasta dónde?, ¿̇con qué consecuencias..?

f) Ecologismo: vuelta a la naturaleza. Progresiva conscientización de los daños causados por años al ambiente natural. Defensa mundial de las especies en peligro de extinción, aun por encima de la seguridad y atención de las necesidades de grupos humanos minoritarios o en situaciones de riesgo.

Si se concede la mayor importancia a "lo natural" (entendiendo por esto lo material) con independencia o en detrimento de lo espiritual, ¿no estará la especie humana en peligro de extinción..?

${ }^{16}$ TABA, H., op.cit., p.66-67. 


\section{LAS GRANDES TENDENCIAS MARCADAS POR LA UNESCO}

Sobre la base del diagnóstico de la situación mundial, la UNESCO promueve desde las últimas décadas del siglo XX, tres grandes tendencias, agregando después una cuarta:

- Aprender a aprender (conocimientos).

- Aprender a hacer (habilidades).

- Aprender a ser (actitudes: valores y virtudes).

Estas tres áreas del aprendizaje humano deberían dar por resultado, impulsadas de modo equilibrado ese educar para la vida", a partir de un desarrollo armónico de la personalidad.

A esta triada se agregó, como respuesta a los procesos generalizados de globalización:

- Aprender a convivir (relaciones humanas).

El tema de la tolerancia entre grupos humanos, la imperiosa necesidad del trabajo en equipo, la interculturalidad en los consorcios multinacionales de trabajo, etcétera, hacen hoy necesaria la inclusión de la convivencia como parte de curriculum escolar.

«Para cumplir el conjunto de las misiones que le son propias, la educación debe estructurarse en torno a cuatro aprendizajes fundamentales que en el transcurso de la vida serán para cada persona, en cierto sentido, los pilares del conocimiento: aprender a conocer, es decir, adquirir los instrumentos de la comprensión; aprender a hacer, para poder influir sobre el propio entorno; aprender a vivir juntos, para participar y cooperar con los demás en todas las actividades humanas; por último, aprender a ser, un proceso fundamental que recoge elementos de los tres anteriores. Por supuesto, estas cuatro vías del saber convergen en una sola, ya que hay entre ellas múltiples puntos de contacto, coincidencia e intercambio ${ }^{17}$.

${ }^{17}$ DELORS, J., La Educación Encierra un Gran Tesoro., p.91. 


\section{6. ¿QUÉ CARACTERIZA A UN «MODELO EDUCATIVO DE CALIDAD?}

Cuando algo es de calidad, vale mucho; todo lo valioso refleja algún tipo de calidad. La calidad, pues, va en relación directa con los valores.

Son valores aquellas fuentes de perfeccionamiento bumano, desde lo material hasta lo espiritual. Por ello distinguimos como elementos de la cultura, distintas «esferas de valor": valores económicos, físicos, sociales, afectivos, intelectuales, estéticos, morales, religiosos ${ }^{18}$.

Un modelo educativo de calidad refleja un conjunto de valores. Por ello habrá de caracterizarse entre otras cosas por ser:

1) Sólido en valores. "La vida humana es un intercambio entre el hombre y su ambiente. (...) La acción educativa relaciona lo cambiante de cada ambiente con lo permanente de cada valor ${ }^{19}$. Se trata de buscar un modelo educativo que a partir de ideas que reflejen la verdad y actos que den ejemplo de Bien, conduzca al:

2) Desarrollo integral. Es decir, el perfeccionamiento de todas las áreas de la personalidad. Éste es y ha sido el gran propósito o finalidad última, del proceso educativo en sus diferentes niveles y en distintos países.

Ya desde 1966, la UNESCO a través del documento «Recomendación relativa a la situación del personal docente" aprobada por la Conferencia Intergubernamental Especial sobre la Situación del Personal Docente, en cooperación con la OIT ${ }^{20}$ (5-X-1966) especificaba que: "La educación debería tener por objeto desde los primeros años de asistencia del niño a la escuela, el pleno desarrollo de la personalidad humana y el progreso espiritual, moral, social, cultural y económico de la comunidad, así como inculcar un profundo respeto por los derechos humanos y las libertades fundamentales. En relación

\footnotetext{
${ }^{18}$ Cfr. PLIEGO, María., Valores y autoeducación., p.68.

${ }^{19}$ CASTILLO, G., op. cit., p. 25.

${ }^{20}$ Organización Internacional del Trabajo.
} 
con estos valores debería concederse la mayor importancia a la contribución de la educación a la paz, así como la comprensión, la tolerancia y la amistad entre todas las naciones y entre los diferentes grupos raciales o religiosos ${ }^{21}$.

Para que este desarrollo integral apunte a la plenitud humana, deberá estar encabezado por la:

a) Formación de la conciencia moral. Como reflejo de lo que es distintivo de la especie humana: la capacidad de pensar y de decidir sobre la propia vida, lo cual implica necesariamente una:

b) Educación en y para la libertad. De modo que se prepare al educando para asumir su propia vida con responsabilidad, para bien propio y ajeno; por ende:

c) Democrático. Capaz de colocarse en el lugar de los demás, hasta el punto de entender su pensar y vivir su sentir; lo cual exige hacer de la educación un factor de equidad y justicia social, que brinde igualdad de oportunidades para todos los sectores de la sociedad. Esto supone entre otras cosas:

d) Escolaridad de vanguardia. Siempre atenta a aportar al educando lo más nuevo y lo mejor, para encauzarlo a convertirse en un:

e) Forjador del futuro. "La sociedad actual y parece que también la futura se caracteriza principalmente por el cambio, uno de los principales factores del cambio social es la tecnología. Los retos como resultado de lo anterior son:

- ¿Cómo hacer compatibles las dos necesidades crecientes: calidad-cobertura?

- ¿Cómo seguir siendo una persona culta en una sociedad con excesiva especialización?

- ¿Cómo adaptarse al nuevo carácter técnico de la sociedad sin perder libertad?

- ¿Cómo utilizar los medios de comunicación sin que sean un obstáculo para la conducta personal?

${ }^{21}$ UNESCO., Informe Mundial sobre la Educación 1998: los Docentes y la Enseñanza en un Mundo de Mutación., p.23. 
- ¿Cómo dar solución a problemas inéditos?

- ¿Cómo mantener un concepto estable de sí mismo ante el excesivo cambio e incertidumbre?

- ¿Cómo armonizar el protagonismo del cambio con la adaptación necesaria al mismo? ${ }^{22}$.

Se trata de construir un modelo educativo que haga de cada sujeto actor de su propio proceso de perfeccionamiento; nunca receptor pasivo de los cambios que la vida desencadena, sino constructor de su propio futuro.

"Por primera vez en la historia de la humanidad, la educación se emplea conscientemente en preparar a los hombres para tipos de sociedad que todavía no existen.

"Por primera vez en la historia de la humanidad, diversas sociedades comienzan a rechazar muchos de los productos que les ofrece la educación institucionalizada ${ }^{23}$.

Estamos hoy ante un nuevo paradigma en el proceso educativo; se trata más que nunca de educar con visión prospectiva, a través de un modelo integrador, que no pierda de vista el ser y el deber ser del hombre como eje que ilumina un progresivo saber, y enciende un positivo hacer.

El "plus" educativo, el "broche de oro", lo proporciona cada educador y cada educando, con su riqueza axiológica personal e irrepetible, con su creatividad, su eticidad, su criticidad, sus ideales y convicciones, que le hacen ser sujeto y no objeto de las grandes tendencias de la educación a inicio del siglo XXI.

Superarnos, para estar "a la altura" de los niños y jóvenes de hoy, ciudadanos del siglo XXI, es nuestro reto como educadores.

${ }^{22}$ Cfr. CASTILLO, Gerardo., op.cit., p.18-23.

${ }^{23}$ Cfr. UNESCO., 21 Puntos para una Nueva Estrategia de Educación., p.5-6. 


\section{BIBLIOGRAFÍA}

ADISESHIAH, M., "Tendencias y Perspectivas de la Educación", en: AAVV, Sobre el Futuro de la Educación. Hacia el año 2000, Narcea, Madrid, 1990, 342 p.

CASTILLO, G., La educación del futuro, en: AAVV, Lo Permanente y lo Cambiante en la Educación, EUNSA, Pamplona, 1991, 149 p.

CERÓN, S., Un Modelo Educativo para México, Ed. Santillana, México, 1998, 380 p.

DELORS, J., La Educación Encierra un Tesoro, Correo de la UNESCO, México, 1997, 302 p.

LASPAlAS, J., GONZÁlEZ, M., MOLINOS, M., Docencia y formación. Estudios en Honor del Profesor José Luis GonzálezSimancas, EUNSA, Pamplona, 1998, 531 p.

PALIN, P., El contexto global para la garantía de la calidad en la Educación Superior: Actividades Recientes de la OECD. Resumen. En: "Reunión trilateral sobre la Globalización de la Educación Superior y las Profesiones": La Globalización de la Educación Superior y las Profesiones: el Caso de América del Norte, SEP, 1994, 248 p.

PLIEGO, M., Valores y Autoeducación, MiNos, México, 2001, 68 p. SECRETARÍA DE EDUCACIÓN PÚBLICA, Programa Nacional de Educación 2001-2006, México, 2001, 269 p.

TABA, H., Elaboración del Currículo: Teoría y Práctica, Troquel, Buenos Aires, 1990, 662 p.

TORRES SANTOMÉ, J., Globalización e Interdisciplinariedad: el Curriculum Integrado, Morata, Madrid, 1996, 279 p.

UNESCO, 21 Puntos para una Nueva Estrategia de la Educación, Magisterio del Río de la Plata, Argentina, Colección. Magisterio 1, 1995, 47 p.

UNESCO, Informe Mundial sobre la Educación 1998: los Docentes y la Enseñanza en un Mundo de Mutación, Madrid, $174 \mathrm{p}$. 